\title{
Toroidal Trapping Effects in the Surface-Averaged Fokker-Planck SSFPQL Solver
}

\author{
R. Bilato and M. Brambilla \\ MPI for Plasma Physics, EURATOM Association, Boltzmannstr. 2, 85748 Garching, Germany
}

\begin{abstract}
The kinetic equation solved by SSFPQL was obtained by surface-averaging the Fokker-Planck (FP) equation, thus neglecting both the finite width of the ion orbits and the poloidal modulation of their velocity. Owing to the latter effect and depending on the position of the resonance layer, a fraction of trapped particles do not even reach the ion-cyclotron resonance. In addition, since the resonant ions gain perpendicular energy, they tend to accumulate on trapped orbits barely intercepting the resonance. Still neglecting the effects due to the finite width of the orbits, here we discuss the form of the FP equation when the velocity modulation is taken into account. In particular, we examine the implementation of this effect in the quasilinear operator of SSFPQL, and present a few numerical results.
\end{abstract}

Keywords: ICRF, full-wave solver, Fokker-Planck solver, orbit average, zero-orbit-width, surface average PACS: $52.40 . \mathrm{Db}$, 52.50.Qt, 52.55.Fa, 52.65.Ff

\section{INTRODUCTION}

The package TORIC-SSFPQL [1] for numerical simulations of Ion Cyclotron (IC) Heating in Tokamaks is particularly fast. This is due in part to the fact that in SSFPQL the solution of the surface-averaged quasilinear kinetic equation is obtained as a velocity dependent superposition of Legendre polynomials in the velocity pitch-angle. This transforms the equation into a (formally infinite) set of ODEs, which are solved using cubic FEM. This approach, however, is straightforward only because the solved kinetic equation neglects not only the finite width of the ion orbits, but also the poloidal modulation of their parallel velocity, and thus the existence of a population of deeply trapped ions which do not reach the IC resonance. These effects can appreciably influence the heating efficiency, and their importance is expected to increase when the heating is applied off-axis.

We present the form taken by the surface-averaged kinetic equation when these effects are included in the model solved by SSFPQL. Their inclusion greatly complicates the evaluation of the coefficients of the ODEs system to be solved, which must be obtained with great precision to avoid convergence problems in the Legendre summation. To make this possible, we have developed a numerical method based on long-integer arithmetics, which could have interesting applications also in other fields of analysis [2]. At present, only the quasilinear term has been implemented with this new method; thus we present preliminary results in which toroidal trapping in the zero-orbit-width (ZOW) approximation is taken into account in the description of resonant IC interactions, but not in the collisional operator.

\section{ZERO-ORBIT-WIDTH AVERAGED QUASILINEAR FOKKER-PLANCK EQUATION}

To highlight the central assumptions done in the following, we briefly recall the main general steps that starting from the orbit average lead to the ZOW-average, and finally to the surface average of SSFPQL [1]. Orbit averaging reduces the dimensionality of the phase space: it is done by building a coordinate system with the constant of motions $(\mathrm{COM})$, which exist for the considered dynamical system, and completing it with the required number of periodic coordinates; then, by averaging over these periodic coordinates, the final equation is formulated in a reduced phasespace spanned only by the COMs. Often this comes at a price: the averaging procedure is complex or/and the usage of COMs is cumbersome. In tokamak axisymmetric configuration a typical set of COMs are the energy per unit mass, $\varepsilon_{v}=v^{2} / 2$ (for simplicity, here the presence of an electrostatic field is neglected), the magnetic momentum per unit mass $\mu_{v}=v_{\perp}^{2} / 2 B$, and the toroidal momentum per unit mass, $P_{\varphi}=-\Omega_{\mathrm{c}} \Psi_{p} / B+R v_{\|} B_{\varphi} / B$, with $\Omega_{\mathrm{c}}$ the cyclotron frequency, $B$ the confining magnetic field and $B_{\varphi}$ its toroidal component, and $\Psi_{p}$ the poloidal magnetic flux. Hereafter, parallel and perpendicular refer to the direction of the local confining magnetic field. The three periodic coordinates are the toroidal, $\varphi$, poloidal, $\vartheta$, and gyro, $\phi_{v}$, angles. This set of coordinates is finally completed with the sign of $v_{\|}$ 
on the external midplane point, $\sigma_{v}$. If the radial drifts are neglected, any monotonic flux function $\rho$ can be used as COM in place of $P_{\varphi}$, which in turn is conserved only on the bounce/transit time $\left(\tau_{b}\right)$ scale. This is what is couched in the zero-orbit-width expression, since the width of the poloidal projection of the trapped orbits shrink to zero when drifts are neglected. Because of the axisymmetry of the configuration, the average over $\varphi$ is straightforward as is the gyro-average. The average over $\vartheta$, instead, requires some discussion, which is postponed to the next section. In short, the ZOW-averaged quasilinear kinetic equation (KE) can be written in divergence form,

$$
\frac{\partial F_{s}}{\partial t}=\frac{1}{\langle J\rangle} \frac{\partial}{\partial z^{i}}\left[\langle J\rangle\left\langle\mathscr{Q}^{i j}\right\rangle \frac{\partial F_{s}}{\partial z^{j}}\right]+\frac{1}{\langle J\rangle} \frac{\partial}{\partial z^{i}}\left[\langle J\rangle\left(\left\langle\mathscr{C}^{i j}\right\rangle \frac{\partial F_{s}}{\partial z^{j}}+\left\langle\mathscr{F}^{i}\right\rangle F_{s}\right)\right]
$$

where $F_{S}$ is the distribution function of the ions species indexed with " $s$ ", $\langle\ldots\rangle$ stands for the average operation, and $J$ is the Jacobian of the starting coordinate system, namely $\left(\phi_{v}, \vartheta, \varphi, \rho, \varepsilon_{v}, \mu_{v}\right)$. Because of the ZOW approximation, equation (1) is a two-dimensional PDE with $\left(z^{i}\right)=\left(\varepsilon_{v}, \mu_{v}\right)$. However, before averaging, we transform the quasilinear operator [3] to the coordinate system having the same angle variables, but $\left(v, \xi_{\mathrm{eq}}, \rho\right)$ as COMs, where $\xi=v_{\|} / v$ : this is the set of COMs used in SSFPQL [1]. Hereafter, the subscript "eq" stays for the value at the external midplane point, through which all the particles transit. In these coordinates the $\mathrm{ZOW}$-averaged quasilinear operator is

$$
\langle\mathscr{D}\rangle=\left(\begin{array}{cc}
\left\langle D_{\mathrm{ql}}\right\rangle & -\frac{\xi_{\mathrm{eq}}}{v}\left\langle D_{\mathrm{ql}} \frac{B_{\mathrm{eq}} / B-1+\xi_{\mathrm{eq}}^{2}}{\xi_{\mathrm{eq}}^{2}}\right\rangle \\
-\frac{\xi_{\mathrm{eq}}}{v}\left\langle D_{\mathrm{ql}} \frac{B_{\mathrm{eq}} / B-1+\xi_{\mathrm{eq}}^{2}}{\xi_{\mathrm{eq}}^{2}}\right\rangle & \left(\frac{\xi_{\mathrm{eq}}}{v}\right)^{2}\left\langle D_{\mathrm{ql}}\left(\frac{B_{\mathrm{eq}} / B-1+\xi_{\mathrm{eq}}^{2}}{\xi_{\mathrm{eq}}^{2}}\right)^{2}\right\rangle
\end{array}\right)
$$

and the diffusion and friction parts of the linearized collisional operator are

$$
\langle\mathscr{C}\rangle=\left(\begin{array}{cc}
\frac{\Psi_{c}(v)}{2 v} & 0 \\
0 & \frac{\Theta_{c}(v)}{2 v}\left(1-\xi_{\mathrm{eq}}^{2}\right)\left\langle\frac{B}{B_{\mathrm{eq}}}\left(\frac{B}{B_{\mathrm{eq}}} \frac{\xi_{\mathrm{eq}}}{\xi}\right)^{-2}\right\rangle
\end{array}\right) \quad \text { and }\langle\mathscr{F}\rangle=\left(\begin{array}{c}
\Psi_{\tau}(v) \\
0
\end{array}\right) .
$$

The full expressions of $\Psi_{c}(v), \Psi_{\tau}(v)$, and $\Phi_{c}(v)$ are given in [1], whereas the quasilinear diffusion coefficient $D_{\mathrm{ql}}$ is derived in [3], and discussed later. Finally, the ZOW average is defined as

$$
\langle(\ldots)\rangle=\frac{1}{\lambda_{B}} \oint \mathrm{d} \vartheta J_{\mathrm{eq}}(\ldots) \quad \text { with } \quad \lambda_{B}=\oint \mathrm{d} \vartheta J_{\mathrm{eq}} \quad \text { and } \quad J_{\mathrm{eq}}=\frac{B}{B_{\mathrm{eq}}} \frac{\xi_{\mathrm{eq}}}{\xi} R \mathscr{J}_{p}
$$

where the space Jacobian $R \mathscr{J}_{p}$ is given in [3] and $\langle J\rangle=\lambda_{B} v^{2}$. In the KE solved so far by SSFPQL, $v_{\|}$is assumed constant. Therefore the energy conservation requires the constancy also of $v_{\perp}$, i.e. $\xi=\xi_{\text {eq }}$. However, since $\mu_{v}$ involves the fastest time scale of the particle motion, $\mu_{v}$ must also be locally conserved, and this implies the constancy of $B$ on the magnetic surface, i.e. $B=B_{\text {eq. }}$ Briefly, in (2) inside the brackets $\langle\ldots\rangle$ survives only $D_{\mathrm{ql}}$, whereas the average disappears from (3). Moreover, the particle velocity simplifies in the average operator, which becomes a pure geometrical average over the whole magnetic surface. This is the meaning of "surface average", and in the next paragraph we show how to relax it for the quasilinear diffusion coefficient in SSFPQL.

\section{ZOW-AVERAGED QUASILINEAR DIFFUSION COEFFICIENT}

We start from the gyro-averaged quasilinear diffusion coefficient derived in [3], and already averaged over $\varphi$

$$
\left\langle D^{i j}\right\rangle=\frac{Z^{2} e^{2}}{2 m^{2}} \operatorname{Re}\left\{\sum_{n_{\varphi}} \int \mathrm{d} \kappa_{1} \int \mathrm{d} \kappa_{2} e^{i\left(\kappa_{2}-\kappa_{1}\right) \rho} \sum_{m_{1}, m_{2}} \sum_{p}\left[\frac{1}{\lambda_{B}} \oint \mathrm{d} \vartheta J_{\mathrm{eq}} \Xi^{\alpha_{i j}}\left(\xi_{\mathrm{eq}}, \vartheta\right) e^{i\left(m_{2}-m_{1}\right) \vartheta} \Delta^{*}\left(p \mid \vec{k}_{1}\right) \int_{-\infty}^{t} \mathrm{~d} t^{\prime} e^{i \chi_{p}\left(\vec{k}_{2} \mid t, t^{\prime}\right) / \eta} \Delta^{\prime}\left(p \mid \vec{k}_{2}\right)\right]\right\}
$$

with $\alpha=\left(\begin{array}{cc}0 & 1 \\ 1 & 2\end{array}\right), \vec{k}=\left(m, n_{\varphi}, \kappa\right)$, and

$$
\begin{gathered}
\Delta(p \mid \vec{k})=\frac{\sqrt{1-\xi^{2}}}{\sqrt{2}}\left[J_{p-1}\left(v_{k} \sqrt{1-\xi^{2}}\right) \mathscr{E}_{+}(\vec{k}) e^{-i \delta_{k}}+J_{p+1}\left(v_{k} \sqrt{1-\xi^{2}}\right) \mathscr{E}_{+}(\vec{k}) e^{-i \delta_{k}}\right], \quad v_{k}=\frac{k_{\perp} v_{\mathrm{th}}}{\Omega_{\mathrm{c}}}, \quad \delta_{k}=\tan ^{-1} \frac{k_{\delta}}{\kappa} \\
\chi_{p}\left(\vec{k} \mid \tau, \tau^{\prime}\right)=\int_{\tau^{\prime}}^{\tau} \mathrm{d} \tau^{\prime \prime}\left(\left(m+q n_{\varphi}\right) \frac{\dot{\vartheta}\left(\tau^{\prime \prime}\right)}{\omega}+p \frac{\Omega_{\mathrm{c}}\left(\tau^{\prime \prime}\right)}{\omega}-1\right), \quad \eta=\frac{v}{q R_{\mathrm{ma}}} \frac{1}{\omega}, \quad \Xi\left(\xi_{\mathrm{eq}}, \vartheta\right)=-\frac{\xi_{\mathrm{eq}}}{v} \frac{b_{\mathrm{eq}}-1+\xi_{\mathrm{eq}}^{2}}{\xi_{\mathrm{eq}}^{2}}
\end{gathered}
$$



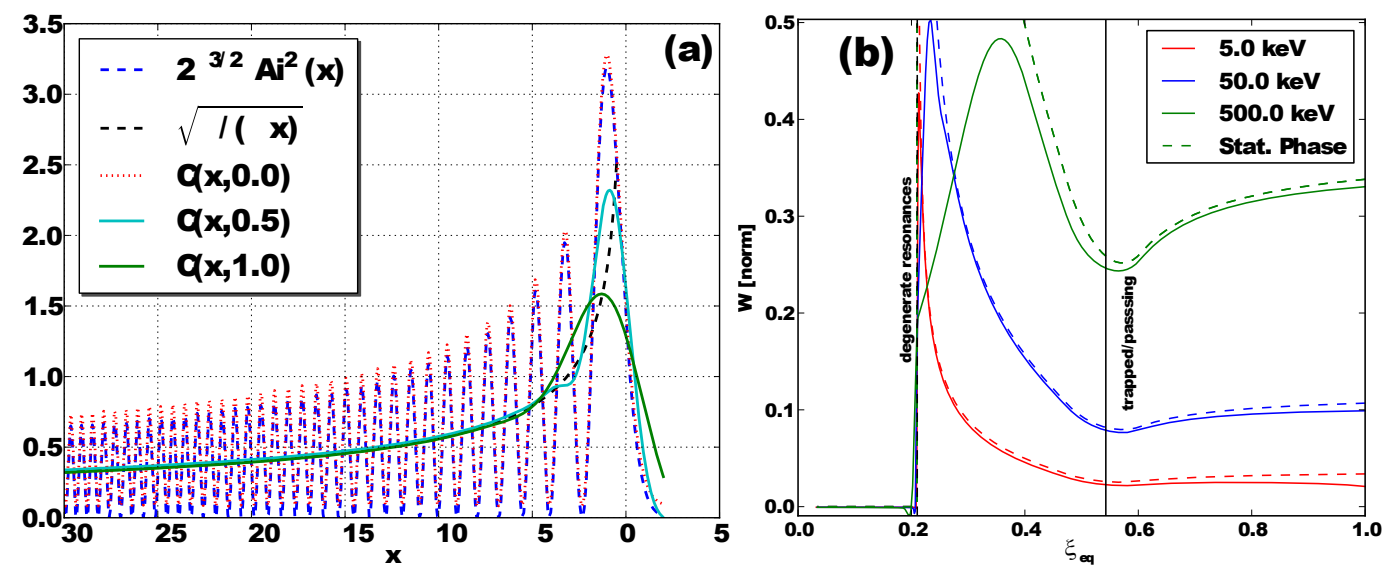

FIGURE 1. (a) Catto-Myra function (7) for different values of the decorrelation parameter $\sigma$. The SP and AF asymptotic solutions are also shown for comparison. (b) Resonance integral $\mathscr{W}=C\left(\xi_{\mathrm{eq}}, \sigma\right) / \tau_{b}$ for three values of the energies. For comparison, the SP approximation is shown in dashed lines. The separation between trapped/passing and resonating/not-resonating particles is shown with vertical solid lines.

where $\mathscr{E}_{ \pm}$are the rotating components of the rf electric fields, $J_{p}(x)$ are the Bessel functions of the first kind, $q$ is the safety factor, and $\delta$ indicates the component along the diamagnetic direction. Upon approximating $\dot{\vartheta} \approx v_{\|} / q R$ with $R(\rho, \vartheta)=R_{\mathrm{ma}} \mathrm{R}(\rho, \vartheta)$ the distance from the torus axis and "ma" for the values on the magnetic axis, the time integral in (5) can be transformed into an integral over $\vartheta$, like the second integral in (5) coming from the formal solution of the Vlasov equation. By splitting the phase $\chi_{p}\left(\vec{k} \mid \tau, \tau^{\prime}\right)=\chi_{p}(\vec{k} \mid \tau, 0)-\chi_{p}\left(\vec{k} \mid \tau^{\prime}, 0\right)$, and by keeping only the contribution from the last bounce/transit period in the second integral (advocating intrinsic and/or extrinsic decorralations between the contributions from nearest periods), the two integrals in (5) can be dealt on the same footing. Since typically $\eta \ll 1$, the integrands are characterized by a slow and a rapidly oscillating components, and the resulting cancellations due to the latter are mitigated only in the neighborhood of stationary-phase points $\tau_{\mathrm{sp}}$, i.e. $\partial \chi_{p} /\left.\partial \tau\right|_{\tau_{\mathrm{sp}}}=0$. If $\tau_{\mathrm{sp}}$ are well separated (secant resonances), the stationary-phase (SP) approximation can be applied, otherwise the interference of the transit through two close resonances (degenerate), is captured with the squared Airy function (AF) [4]. To prescribe a smooth transition between these two asymptotic regimes, we use the heuristic model proposed by Catto and Myra [5] and justified in presence of decorrelation mechanisms, such as collisions. In brief, the time integrals are approximated with

$$
\frac{1}{\tau_{b}} \oint \mathrm{d} t^{\prime} f\left(t^{\prime}\right) e^{-i \chi\left(t^{\prime}\right)} \approx \sum_{\tau_{\text {icr }}} f\left(\tau_{\text {icr }}\right) \mathrm{C}\left(g\left(\tau_{\text {icr }}, \xi_{\mathrm{e}}\right), \sigma_{c}\right) \quad \text { with } \quad \mathrm{C}(x, \sigma)=\int_{0}^{+\infty} \frac{\mathrm{d} \rho}{\sqrt{\rho}} \cos \left(x \rho+\frac{\rho^{3}}{12}+\frac{\pi}{4}\right) e^{-\sigma \rho^{3}}
$$

where the subscript "icr" stands for the values at the ion-cyclotron resonance, $f(t)$ represents the slow part of the integrands in (5). Decorrelations enter via $\sigma$, which depends at least on the collisional frequency. The Catto-Myra function $\mathrm{C}(x, \sigma)$ is shown in figure (1.a) for a few values of $\sigma$, and for comparison the AF and SP asymptotic solutions are overplotted. For $x \ll-1, \mathrm{C}(x, \sigma)$ matches the SP solution when $\sigma>0$, whereas it overlaps the AF approximation when $\sigma=0$. The major obstacle in matching the SP and AF asymptotic solution is the difference in their arguments. We can derive a convenient expression for $g\left(\tau_{\text {icr }}, \xi_{\mathrm{e}}\right)$ if we assume that the confining magnetic field varies like $B(\rho, \vartheta)=B_{\mathrm{ma}} / \mathrm{R}(\rho, \vartheta)$,

$$
g\left(\tau_{\text {icr }}, \xi_{e}\right)= \begin{cases}\frac{1}{\sqrt{\pi}} \frac{\mathrm{R}_{e}}{\mathrm{R}_{\mathrm{icr}}^{5}}\left(\frac{\partial \mathrm{R} /\left.\partial \vartheta\right|_{\text {icr }}}{2 \eta}\right)^{2}\left(\xi_{\mathrm{deg}}^{2}-\xi_{\mathrm{e}}^{2}\right) & \text { for } \xi_{\mathrm{e}}>\xi_{\mathrm{deg}} \\ \mathrm{R}_{\mathrm{e}}\left(\frac{\mathrm{R}_{\mathrm{e}}}{\mathrm{R}_{\mathrm{icr}}}\right)^{1 / 3}\left(\frac{2}{\eta \partial R /\left.\partial \vartheta\right|_{\text {icr }}}\right)^{2 / 3}\left(1-\xi_{e}^{2}\right)^{1 / 3}\left(\xi_{\mathrm{deg}}^{2}-\xi_{\mathrm{e}}^{2}\right) & \text { for } \quad \xi_{\mathrm{e}}<\xi_{\mathrm{deg}}\end{cases}
$$

A particle with $\xi_{\mathrm{e}}$ equal to $\xi_{\mathrm{deg}}=\sqrt{1-\mathrm{R}_{\mathrm{icr}} / \mathrm{R}_{e}}$ has its banana tips at the icr resonance layer, which intercepts the magnetic surface if $\mathrm{R}_{i} \leq \mathrm{R}_{\mathrm{icr}} \leq \mathrm{R}_{\mathrm{e}}$, with "i" standing for the internal midplane point. An example of the resonance integral $\mathscr{W}=\mathrm{C} / \tau_{b}$ for three values of the particle energy is shown in figure (1.b). In the model (8) we have neglected the Doppler shift in the phase $\chi_{p}$. Thus, the dependence of $\mathscr{W}$ on the particle energy enter only through $\eta$. For $\xi<\xi_{\mathrm{deg}}$, $\mathscr{W}$ goes to zero, since the particle do not resonate with the waves. The hollow around the passing/trapped boundary is due to the increase of $\tau_{b}$ when approaching that border. 

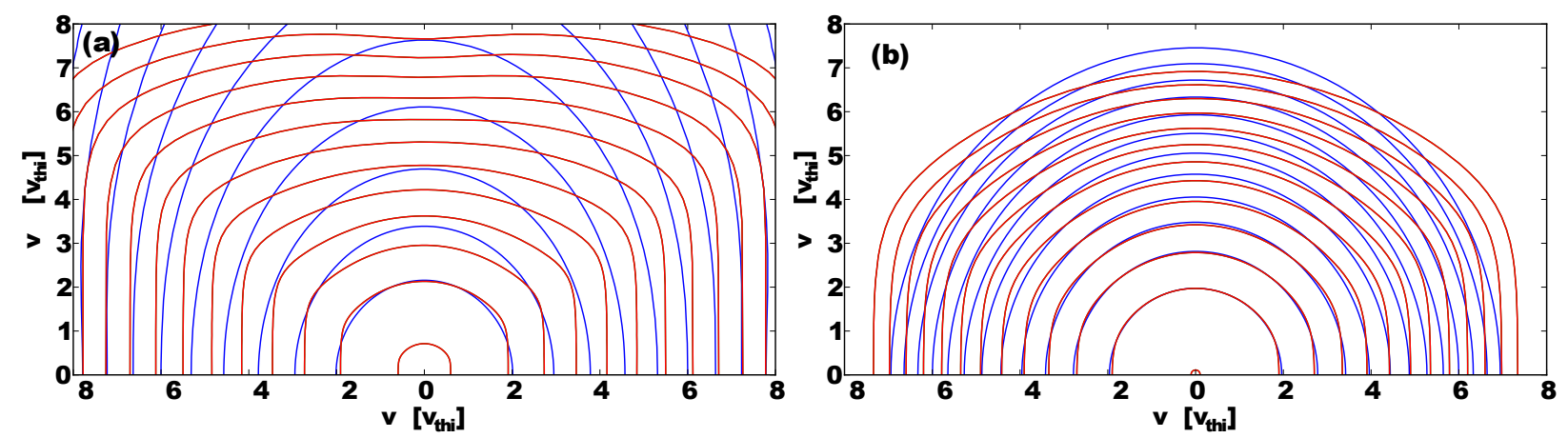

FIGURE 2. Contour plots of the hydrogen (minority) distribution function with (red) and without (blue) trapping effects. (a) At the maximum of absorbed power (about $x / a=0.35$ ) with $\mathrm{R}_{\mathrm{e}}=1.095, \mathrm{R}_{\mathrm{icr}}=1.089, \mathrm{R}_{\mathrm{i}}=0.895, \xi_{\mathrm{deg}}=0.076$, and $\xi_{\text {trp }}=0.428$. (b) At around $x / a=0.5$ with $\mathrm{R}_{\mathrm{e}}=1.131, \mathrm{R}_{\mathrm{icr}}=1.096, \mathrm{R}_{\mathrm{i}}=0.850, \xi_{\mathrm{deg}}=0.175$, and $\xi_{\text {trp }}=0.499$.

\section{NUMERICAL RESULTS}

The numerical scheme of SSFPQL is based on the expansion of the solution in Legendre polynomials w.r.t. $\xi_{\text {eq }}{ }^{-}$ dependence, which are eigenfunctions of the pitch angle part of the collisional diffusion operator. This is possible because the dependence of the surface-averaged $D_{\mathrm{ql}}$ on $v$ and $\xi_{\mathrm{eq}}$ can be separated thanks to the multiplication theorem of Bessel functions. In doing so, the integration of the kinetic equation over $\xi_{\mathrm{eq}}$ is reduced to integrals involving only Legendre polynomials, which can be calculated recursively. The key dodge of the algorithm is that all these integrals can be calculated with machine precision by means of software for long-integer arithmetics [1]. Recently we have shown that this stratagem can be extended to general $\xi_{\text {eq }}$-dependences of $D_{\mathrm{ql}}$ [2]. However, the $v$ - and $\xi_{\text {eq-dependence }}$ of the resonance integrals in the ZOW-averaged $D_{\mathrm{ql}}$ is entangled in such a way that the separation of variables is not anymore feasible. In absence of an expedient, we consider a constant energy to estimate $\eta$, and for the cases considered here the results are not so sensitive to the chosen value if it ranges from the thermal energy to a few tens times it. As an illustrative example, we have considered the conventional minority heating scenario of hydrogen in deuterium plasma, where the IC resonance layer intercepts the midplane at $x / a=0.4$ on the 1.f.s. The contour plot of the hydrogen distribution function (df) at about the maximum of rf power absorption is shown in figure (2.a): for comparison the blue lines are obtained neglecting the trapping effects. As it is well known, the trapping effects reduce considerably the df anysotropy. In addition, we have considered as reference energy for $\eta$ the thermal energy and twenty times it, but the differences are no visible in figure (2.a). Finally, the df in figure (2.b) refers to a more off-axis position $(x / a \approx 0.5)$ where the absorbed power is much less. The comparison between figures (2.a) and (2.b) confirms a common understanding that the higher the power absorbed is, the more visible the trapping effects are.

\section{CONCLUSIONS}

In [2] we proved the feasibility of accounting for the $v_{\|}$modulation in SSFPQL code, and here we have outlined the physical model which takes advantage of this extension. Presently in progress there are the revising of the power balance and the updating of the interface with TORIC, which now requires the mapping of the distribution function from the external midplane point to the position of the IC resonance. Finally, the ZOW-averaged collisional operator must be included in the physical model of SSFPQL. These issues will be discussed in the near future.

Acknowledgement. We are grateful to O. Maj and V. Belmondo for many useful discussions.

\section{REFERENCES}

1. M. Brambilla, and R. Bilato, Nuclear Fusion 49, 085004 (2009).

2. R. Bilato, M. Brambilla, and Z. Jiang, Journal of Physics: Conference Series 401, 012001 (2012).

3. M. Brambilla, Nuclear Fusion 47, 175-180 (2007).

4. I. B. Bernstein, and D. C. Baxter, Physics of Fluids 24. 108-126 (1981).

5. P. J. Catto, and J. R. Myra, Physics of Fluids B: Plasma Physics 4, 187-199 (1992). 